\title{
SSSPM J1102-3431: A probable new young brown dwarf member of the TW Hydrae Association *
}

\author{
R.-D. Scholz ${ }^{1}$, M. J. McCaughrean ${ }^{1,2}$, H. Zinnecker ${ }^{1}$, and N. Lodieu ${ }^{1,3}$ \\ 1 Astrophysikalisches Institut Potsdam, An der Sternwarte 16, 14482 Potsdam, Germany \\ e-mail: [rdscholz;hzinnecker] @aip.de \\ ${ }^{2}$ University of Exeter, School of Physics, Stocker Road, Exeter EX4 4QL, UK \\ e-mail: mjm@aip.de; mjm@astro.ex.ac.uk \\ 3 University of Leicester, Department of Physics \& Astronomy, University Road, Leicester LE1 7RH, UK \\ e-mail: nl41@star.le.ac.uk
}

Received 18 November 2004 / Accepted 9 December 2004

\begin{abstract}
We have used archival survey data, most importantly from the SuperCOSMOS Sky Surveys, to derive accurate proper motions and thus confirm the membership of two previously announced isolated brown dwarfs in the nearby, 10 Myr old TW Hydrae Association (TWA), 2MASSW J1207334-393254 and 2MASSW 1139511-315921 (Gizis 2002), and to discover a new substellar candidate. This new object, SSSPMJ1102-3431, has extremely red optical, optical-to-infrared, and nearinfrared colours $\left(R-I=3.2, I-J=3.4, J-K_{\mathrm{s}}=1.15\right)$, while low-resolution not only classifies it as a late-type (M8.5) object, but also shows spectral signatures of low gravity as expected for a young brown dwarf. If it is a true TWA member, brown dwarf cooling models predict its mass to be $\sim 20 M_{\text {Jup }}$. SSSPM J1102-3431 lies only $\sim 12$ arcmin away from the T Tauri star TW Hya and their proper motions agree to within the errors, and thus it is possible that the two objects form an extremely wide binary system, with a separation of $\sim 40000 \mathrm{AU}$ at $d=56 \mathrm{pc}$.

We have also obtained improved proper motions for SSS 120727.4-324700 and SSS 101726.7-535428, recent additions to the list of TWA members (Song et al. 2003). The relatively large proper motion of the latter object is consistent with the suggested proximity $(d \sim 22 \mathrm{pc})$ of this lowest-mass stellar representative of the TWA.
\end{abstract}

Key words. astrometry and celestial mechanics: astrometry - surveys - stars: kinematics - stars: low-mass, brown dwarfs open clusters and associations: individual: TW Hydrae

\section{Introduction}

The TW Hydrae association (TWA) was identified and named by Kastner et al. (1997) as a group of associated T Tauri stars at a distance of about $50 \mathrm{pc}$ from the Sun. With its originally estimated age of about $20 \mathrm{Myr}$, the association has been regarded as the closest known region of recent star formation. In common with other young associations and moving groups which have been identified during the past seven years in surprising proximity to the Sun $(d<100 \mathrm{pc})$ and without corresponding molecular clouds, the TWA has been a target of many investigations (see Zuckerman \& Song 2004 for a review).

The TWA members share common signatures of youth: they show high X-ray fluxes, large lithium abundances, and strong chromospheric activity (see, e.g., Kastner et al. 1997; Webb et al. 1999; Zuckerman et al. 2001; Song et al. 2003 (hereafter SZB03)). The stars are distributed over a large area of the sky ( $\alpha$ between $10^{\mathrm{h}}$ and $13^{\mathrm{h}}, \delta$ from $-25^{\circ}$ to $-55^{\circ}$ )

^ Based on archival data from the SuperCOSMOS Sky Surveys, 2MASS, and DENIS, and on spectroscopic observations with the ESO 3.6-m telescope (ESO 072.C-0630). and are correspondly spread over several tens of parsecs along the line of sight. Their proper motions $\left(\mu_{\alpha} \cos \delta\right.$ from -33 to $-122 \mathrm{mas} / \mathrm{yr}$ and $\mu_{\delta}$ from -10 to $-43 \mathrm{mas} / \mathrm{yr}$ ) and radial velocities (between +6 and $+17 \mathrm{~km} \mathrm{~s}^{-1}$ ) are similar, indicating common space motions (Reid 2003).

More than 25 TWA members, many of which are actually binaries and multiples, are currently known (Zuckerman \& Song 2004). These are mainly $\mathrm{K}$ and $\mathrm{M}$ dwarfs with photometrically derived distances of between about $20 \mathrm{pc}$ and $130 \mathrm{pc}$. Accurate HIPPARCOS parallaxes (ESA 1997) exist for only two of these later-type stars, HD 98800 (K5) and TW Hya itself (K7), as well as the only two early members of the association, HR 4796 (A0) and HD 102458 (G5): the distances lie between 47 and $104 \mathrm{pc}$.

Given the proximity and youth of the association, it has become a key target of searches for very low-mass stars, brown dwarfs, and planets as companions to the higher-mass members, since they should be relatively bright and wellseparated in angular terms from their primaries. In addition, there have been searches for new, isolated, low-mass members which would serve as good targets for further 
companion searches. For example, Webb et al. (1999) identified new $\mathrm{T}$ Tauri star members in the TWA, found a brown dwarf companion to a previously known TWA member (TWA 5B), and suggested that the TWA may be as young as $10 \mathrm{Myr}$. The first isolated brown dwarf candidate members of the TWA, 2MASSW J1207334-393254 and 2MASSW J1139511-315921 (hereafter 2MASS 1207 and 2MASS 1139, respectively) were discovered by Gizis (2002) in a colour-based search using data from the Two-Micron All Sky Survey (2MASS; Cutri et al. 2003). All three of these candidate brown dwarfs have subsequently been confirmed as true substellar TWA members by high-resolution spectroscopy (Mohanty et al. 2003).

In this letter, we present results from a proper-motion based study of two previously known and one new brown dwarf member of the TWA. We present follow-up observations of the new candidate, SSSPM J1102-3431 (hereafter SSSPM 1102), and discuss its properties: it appears to be one of the lowest-mass substellar members of the TWA found to date.

\section{Proper motion survey}

We have been conducting a high proper motion survey of the southern sky based on archival data from the SuperCOSMOS Sky Surveys (SSS; Hambly et al. 2001a,b,c) in the optical and 2MASS (Cutri et al. 2003) in the near-infrared. One focus of our work has been to try and push down to a proper motion limit of $\sim 50$ mas/yr for faint $(R>13)$ red objects likely to be nearby dwarfs, without too large a kinematic selection bias. A potentially major source of photometric contamination would be distant $(>10 \mathrm{kpc})$ red giants, but these are readily eliminated by the proper motion criterion, since the implied tangential velocities of $>2000 \mathrm{~km} \mathrm{~s}^{-1}$ would exceed the Galactic escape velocity. We have applied different search strategies to the SSS and 2MASS data in previous studies (see, e.g., Scholz \& Meusinger 2002; Scholz et al. 2003), and next we describe the method used to identify the new candidate, SSSPM 1102.

We started with the 2MASS, selecting relatively bright $(J<$ 14) southern sky objects out of the Galactic plane $\left(|b|>20^{\circ}\right.$, but excluding the Magellanic Clouds) whose nominal optical counterpart tabulated in the 2MASS data base lay at a separation of 1-5 arcsec. These optical counterpart identifications were drawn from the USNO A2.0 catalogue (Monet et al. 1998). Given the epoch differences between the 2MASS and A2.0 observations, we proceeded from the presumption that the astrometric mismatch may often be due to the object having a high proper motion. In order to focus on brown dwarfs, we also required that the nominal counterparts show a large optical-toinfrared colour of $R-K_{\mathrm{s}}>6$ or $B-K_{\mathrm{s}}>8$. These selection criteria resulted in a list of about 1000 objects which were then checked in the multi-colour, multi-epoch SSS data archives to see if they were indeed extremely red proper motion objects.

Once a candidate was identified, an accurate proper motion was determined using all available SSS positions (including those from overlapping plates), the 2MASS position, and additional epoch positions from the DEep Near-Infrared Survey (DENIS; Epchtein et al. 1997), if available. The resulting accuracy of about $10 \mathrm{mas} / \mathrm{yr}$ allowed us not only to investigate "classical" high proper motion objects with $\mu>0.18 \mathrm{arcsec} / \mathrm{yr}$ (i.e., the lower limit in the New Luyten Two Tenths (NLTT) catalogue; Luyten 1979-80), but also to find members of nearby associations with mean proper motions only about half as large as the NLTT lower limit. These sources were then carried forward for spectroscopic follow-up observations.

\section{TWA brown dwarf candidates}

The search strategy outlined above enabled us to recover several sources in the TWA from the study of Gizis (2002), including one of the two M8.0 brown dwarfs, 2MASS 1139. The second BD in his sample, 2MASS 1207, was listed without an optical counterpart in the 2MASS data base and was therefore not identified by our initial search: we added it to our sample by hand afterwards. In addition, however, we did find a new object, SSSPM 1102, with similar proper motion and colours not previously identified by Gizis (2002) or by other surveys. The reason, why Gizis (2002) missed SSSPM 1102 in his survey, remains unclear, since this object seems to satisfy his search criteria applied to the 2MASS data base. The SSS, 2MASS, and DENIS colours for SSSPM 1102 are very similar to those of 2MASS 1139, the redder of the two previously known M8.0 brown dwarfs (Table 1), leading us to suspect a similarly late spectral type.

Table 1 also lists accurate proper motion measurements for the two previously known isolated TWA brown dwarfs (Gizis 2002) and for the new candidate. All three objects have proper motions consistent with the proper motions of other known members of the TWA (see, e.g., SZB03). Membership of 2MASS 1139 in the TWA was not clear from the rough proper motion measurement by Gizis (2002) and was also doubted by Reid (2003), but our more accurate proper motion measurements do now clearly support its TWA membership. In the lower part of Table 1, we also show new, more accurate proper motions for two stellar TWA candidate members, including an M5 dwarf at only 22 pc from the Earth (SZB03).

\section{Spectroscopy}

Low-resolution $(R \sim 600)$ optical spectroscopy was obtained on 16 March 2004 for a sample of extremely red, high proper motion objects, including SSSPM 1102 and the two comparison objects shown in Fig. 1. EFOSC2 on the ESO 3.6-m telescope was used in service mode (ESO programme 072.C-0630), with grism\#5 providing a wavelength coverage 5200-9350 $\AA$ at a FWHM resolution of $\sim 12.8 \AA$. The exposure times were $1800 \mathrm{~s}, 480 \mathrm{~s}$, and $360 \mathrm{~s}$ for SSSPM 1102, LP 944-20, and DENIS-P J104814.7-395606.1 (hereafter DENIS 1048), respectively. The spectral extraction, wavelength and flux calibration were performed using standard MIDAS routines.

Figure 1 shows the spectrum of SSSPM 1102 compared with those for two other very late-type $\mathrm{M}$ dwarfs of different ages. One is the well-known proper motion object LP 944-20 (Luyten 1979-80), classified as an M9.5 (Reid et al. 2002) field brown dwarf with an intermediate age of $\sim 475-650 \mathrm{Myr}$ (Tinney 1998). The other is the nearby M9.0 field dwarf 
Table 1. Astrometry and photometry from SSS, 2MASS and DENIS for two previously known TWA brown dwarfs, the new candidate brown dwarf SSSPM 1102 (upper section), and improved proper motions and photometry for two stellar TWA members proposed by SZB03.

\begin{tabular}{|c|c|c|c|c|c|c|c|c|c|c|}
\hline \multirow[t]{2}{*}{ Name } & $\alpha, \delta$ & Epoch & $\mu_{\alpha} \cos \delta$ & $\mu_{\delta}$ & $B_{J}$ & $R$ & \multirow{2}{*}{$\begin{array}{c}I \\
\text { DENIS } \\
\end{array}$} & $J$ & \multirow{2}{*}{$\begin{array}{c}H \\
\text { 2MASS }\end{array}$} & \multirow[t]{2}{*}{$K_{\mathrm{s}}$} \\
\hline & $\mathrm{J} 2000$ & & \multicolumn{2}{|c|}{$\mathrm{mas} / \mathrm{yr}$} & \multicolumn{2}{|c|}{ SSS } & & & & \\
\hline SSSPM $1102^{a}$ & $110209.83-343035.5$ & 1999.236 & $-82 \pm 12$ & $-12 \pm 06$ & 22.168 & 19.573 & 16.198 & 13.034 & 12.356 & 11.887 \\
\hline 2 MASS $1139^{b}$ & $113951.13-315921.4$ & 2000.041 & $-93 \pm 05$ & $-31 \pm 10$ & 21.729 & 19.090 & 15.829 & 12.686 & 11.996 & 11.503 \\
\hline $2 \mathrm{MASS} 1207^{c}$ & $120733.47-393254.0$ & 1999.334 & $-78 \pm 11$ & $-24 \pm 09$ & 21.432 & 19.064 & 15.875 & 12.995 & 12.388 & 11.945 \\
\hline SSS $101726^{d}$ & $101726.89-535426.5$ & 2001.110 & $-176 \pm 07$ & $-22 \pm 08$ & 15.088 & 12.367 & 10.493 & 8.554 & 8.085 & 7.689 \\
\hline SSS $120727^{e}$ & $120727.38-324700.3$ & 1999.247 & $-68 \pm 04$ & $-23 \pm 04$ & 13.819 & 12.196 & 10.134 & 8.618 & 8.025 & 7.751 \\
\hline
\end{tabular}

Notes:

Coordinates are taken from 2MASS, which provided accurate coordinates at the most recent epoch. SSS magnitudes are mean values. The proper motions are mainly based on the multi-epoch SSS data with a typical baseline of 18 years and on additional recent epoch observations extending the time baseline by about 5 years. Full names, spectral types and individual notes are given below.

${ }^{a}$ SSSPM J1102-3431 (M8.5; this work); $\mu$ from 1 2MASS, 1 DENIS, and 7 SSS positions; SSS $I=16.406$.

${ }^{b}$ 2MASSW J1139511-315921 (M8; Gizis 2002); $\mu$ from 1 2MASS, 1 DENIS, and 4 SSS positions; SSS $I=15.702$.

c 2MASSW J1207334-393254 (M8; Gizis 2002); $\mu$ from 1 2MASS, 1 DENIS, 1 Chandra, and 4 SSS positions; SSS $I=16.193$.

${ }^{d}$ SSS 101726.7-535428 (M5; SZB03); $\mu$ from 1 2MASS, 2 DENIS, and 4 SSS positions; SSS $I=9.659$.

${ }^{e}$ SSS 120727.4-324700 (M1; Zuckerman \& Song 2004, SZB03); $\mu$ from 1 2MASS, 1 DENIS, and 11 SSS positions; SSS $I=10.144$; large differences between individual magnitudes in all SSS bands perhaps due to variability?

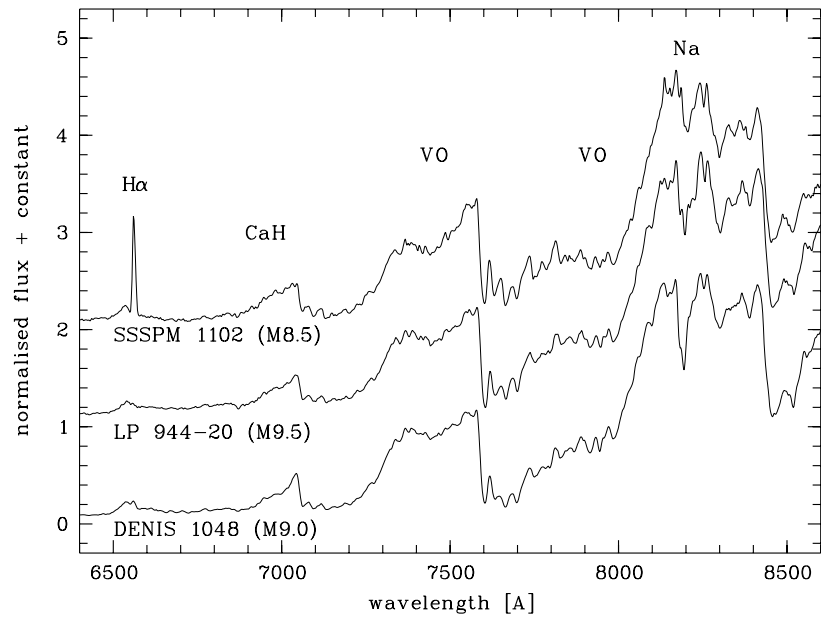

Fig. 1. Optical spectrum for SSSPM 1102 (M8.5, with an assumed age of $\sim 10 \mathrm{Myr}$ as discussed in the text), compared with spectra for two older, nearby field dwarfs, the $\sim 500 \mathrm{Myr}$ old brown dwarf LP $944-20$ (M9.5) and the $\sim 1-2$ Gyr old DENIS 1048 (M9.0). Note the trend toward stronger $\mathrm{CaH}, \mathrm{Na}$, and weaker VO with age. For SSSPM 1102 we measured the largest VO-a and VO-b indices (Kirkpatrick et al. 1999): $\mathrm{VO}-\mathrm{a}=1.31$ and $\mathrm{VO}-\mathrm{b}=1.74$, compared to 1.20 and 1.57 for LP 94420 and 1.14 and 1.51 for DENIS 1048.

DENIS 1048 (Delfosse et al. 2001), with an age of 1-2 Gyr (Neuhäuser et al. 2002; Fuhrmeister \& Schmitt 2004). We have classified SSSPM 1102 as M8.5 based on its PC3 index (Martín et al. 1999) value of 1.92, and based on a direct comparison of the three spectra shown in Fig. 1.

As demonstrated by Gizis (2002), young brown dwarfs such as those in the TWA, show low-gravity features in their spectra (see also McGovern et al. 2004). In common with the spectra shown in Fig. 5 of Gizis (2002), we find that SSSPM 1102 exhibits stronger VO and weaker $\mathrm{CaH}$ and
$\mathrm{Na}$ absorption when compared to older late-M dwarfs: there is a clear trend in the change of these low-gravity features with the age of the objects shown in Fig. 1. SSSPM 1102 also shows a strong $\mathrm{H} \alpha$ emission line (equivalent width $10 \AA$ ) and thus combined with the low-gravity tracers, it appears unequivocal that SSSPM 1102 is a young source.

\section{Discussion and outlook}

All of the available information for SSSPM 1102, including its accurate proper motion, photometry, and spectroscopy, lead to the conclusion that it is very likely a member of the young, nearby TW Hydrae association. If so, then it should be one of the lowest-mass substellar TWA representatives discovered so far. By comparison with the pre-main sequence (PMS) evolutionary models of Chabrier et al. (2000), Gizis (2002) concluded that both 2MASS 1207 and 2MASS 1139 have masses of $\sim 0.025 M_{\odot}$ or $\sim 25 M_{\text {Jup }}$. Lowrance et al. (1999) also suggested a mass of $\sim 0.02 M_{\odot}$ for TWA $5 \mathrm{~B}$, which they classified as M8.0-8.5 based on its colour. SSPM 1102 has rather similar colours to 2MASS 1207 and 2MASS 1139, and our spectral classification of SSSPM 1102 is half a spectral type later than those two M8 dwarfs, and thus it seems very likely that its mass would also be at or below $25 M_{\text {Jup }}$.

The lack of an accurate age and distance for SSSPM 1102 makes it difficult to be more precise about the mass estimate. However, we note that SSSPM 1102 lies only about 12 arcmin away from the eponymous T Tauri star TW Hya. The proper motion given for TW Hya in the HIPPARCOS catalogue (ESA 1997) is $(-67,-12) \mathrm{mas} / \mathrm{yr}$, whereas the Tycho-2 catalogue (Høg et al. 2000) lists $(-73,-17)$ mas/yr. If we opt for the Tycho- 2 proper motion based on its longer time baseline, we see that it is shared by SSSPM 1102 to within the errors. Thus it is possible that not only is SSSPM 1102 a probable member of the TWA, but it may also be a wide binary 
companion to TW Hya itself, with the measured angular separation corresponding to a projected separation of $\sim 40000 \mathrm{AU}$ at the HIPPARCOS distance of $\sim 56 \mathrm{pc}$ to TW Hya (ESA 1997).

If we now take this distance of $56 \mathrm{pc}$ and assume an age of $10 \mathrm{Myr}$ (Webb et al. 1999), we can compare the photometric properties of SSSPM 1102 with the PMS models. Examining the models of Baraffe et al. (1998), we find a (probably spuriously) good fit (to within $\pm 0.1 \mathrm{mag}$ ) to the $M_{I}, M_{J}, M_{H}$, and $M_{K_{\mathrm{s}}}$ absolute magnitudes of SSSPM 1102 is given by a $\sim 20 M_{\text {Jup }}$ brown dwarf. The photographic $M_{R}$ magnitude is too low by $\sim 1$ mag with respect to the same model, but this is in a non-standard filter system and in a region where the model atmospheres are not too well characterised. The corresponding effective temperature $(\sim 2500 \mathrm{~K})$, luminosity $\left(\log L / L_{\odot} \sim\right.$ $-2.70)$, and surface gravity $(\log g \sim 4$ ) tabulated for a $10 \mathrm{Myr}$, $20 M_{\text {Jup }}$ source by Baraffe et al. (1998) are also well-matched by the model predictions of Burrows et al. (1997), adding further confidence to the result.

Although there is clearly room for degeneracy in this process, given that we actually know neither the age nor distance, SSSPM 1102 appears to be a very good candidate low-mass brown dwarf member of the TWA. More detailed observations are needed to confirm its membership, in particular higher resolution spectroscopy to search for lithium absorption and to obtain its radial velocity. It should also be placed on a trigonometric parallax programme so that an accurate distance and space motion can be determined. Furthermore, as a young brown dwarf candidate, SSSPM 1102 should be studied in more detail in the near- and mid-infrared, in order to establish whether or not it has ongoing accretion as was found for 2MASS 1207 (Mohanty et al. 2003; Sterzik et al. 2004), and to search for possible planetary-mass companions (cf. Chauvin et al. 2004).

Finally, we have used all available SSS, 2MASS, and DENIS data to determine accurate proper motions for two other new TWA members recently found in the SSS by SZB03. They did not report a proper motion for SSS 120727, but we find it to be very similar to other bona fide TWA members (Table 1). On the other hand, the proper motion we have computed for SSS 101726 of $(-176,-22) \pm(7,8) \mathrm{mas} / \mathrm{yr}$ is significantly larger than that given by SZB03 (note also that in their text, Sect 2.1 , they give the wrong sign for $\mu_{\alpha} \cos \delta$, i.e., +145 instead of $-145 \mathrm{mas} / \mathrm{yr}$ ). This new proper motion is roughly twice as large as that for other confirmed TWA members and is therefore consistent with this M5 dwarf being very nearby ( 22 pc) as reported by SZB03. If confirmed as a true TWA member, SSS 101726 would be the nearest and lowest-mass stellar representative of the TWA, although trigonometric parallax and radial velocity measurements are lacking at present.

Acknowledgements. This research is based on data from the SuperCOSMOS Sky Surveys at the Wide-Field Astronomy Unit of the Institute for Astronomy, University of Edinburgh. We have also used data products from the Two Micron All Sky Survey, which is a joint project of the University of Massachusetts and the Infrared Processing and Analysis Center/California Institute of Technology, funded by the National Aeronautics and Space Administration and the National Science Foundation, and from the DEep NearInfrared Survey (DENIS). We would like to thank the observers, Linda Schmidtobreick and Ivo Saviane, and the EFOSC2 support astronomer, Gaspare Lo Curto, for their efforts in carrying out our spectroscopic observations in service mode on the ESO 3.6-m telescope. Finally, we thank Isabelle Baraffe and Gilles Chabrier for helpful discussions, and we would also like to thank the referee, B. Zuckerman, for his useful comments.

\section{References}

Baraffe, I., Chabrier, G., Allard, F., \& Hauschildt, P. 1998, A\&A, 337, 403

Burrows, A., Marley, M., Hubbard, W. B., et al. 1997, ApJ, 491, 856

Chabrier, G., Baraffe, I., Allard, F., \& Hauschildt, P. 2000, ApJ, 542, 464

Chauvin, G., Lagrange, A.-M., Dumas, C., et al. 2004, A\&A, 425, L29 Cutri, R. M., Skrutskie, M. F., Van Dyk, S., et al. 2003, The 2MASS All-Sky Catalog of Point Sources, University of Massachusetts and Infrared Processing and Analysis Center (IPAC/California Institute of Technology)

Delfosse, X., Forveille, T., Martín, E. L., et al. 2001, A\&A, 366, L13 Epchtein, N., de Batz, B., Capoani, L., et al. 1997, Messenger 87, 27

ESA 1997, HIPPARCOS and Tycho catalogues, ESA-SP 1200

Fuhrmeister, B., \& Schmitt, J. H. M. M. 2004, A\&A, 420, 1079

Gizis, J. E. 2002, ApJ, 575, 484

Hambly, N. C., MacGillivray, H. T., Read M. A., et al. 2001a, MNRAS, 326, 1279

Hambly, N. C., Irwin, M. J., \& MacGillivray, H. T. 2001b, MNRAS, 326, 1295

Hambly, N. C., Davenhall, A. C., Irwin, M. J., \& MacGillivray, H. T. 2001c, MNRAS, 326, 1315

Høg, E., Fabricius, C., Makarov, V. V., et al. 2000, A\&A, 355, L27

Kastner, J. H., Zuckerman, B., Weintraub, D. A., \& Forveille, T. 1997, Science, 277, 67

Kirkpatrick, J. D., Reid, I. N., Liebert, J., et al. 1999, ApJ, 519, 802

Lowrance, P. J., McCarthy, C., Becklin, E. E., et al. 1999, ApJ, 512, L69

Luyten W. J. 1979-1980, New Luyten Catalogue of Stars with Proper Motions Larger than Two Tenths of an Arcsecond (Minneapolis: Univ. Minnesota), ADC Selected Astronomical Catalogs Vol.1, CD-ROM

Martín, E. L., Delfosse, X., Basri, G., et al. 1999, AJ, 118, 2466

McGovern, M. R., Kirkpatrick, J. D., McLean, I. S., et al. 2004, ApJ, 600,1020

Mohanty, S., Jayawardhana, R., \& Barrado y Navascues, D. 2003, ApJ, 593, L109

Monet, D., Bird, A., Canzian, B., et al. 1998, The USNO-A2.0 Catalogue, US Naval Observatory Flagstaff Station (USNOFS) and Universities Space Research Association (USRA) stationed at USNOFS

Neuhäuser, R., Guenther, E. W., Alves, J., et al. 2002, Astron. Nachr., 323,447

Reid, I. N. 2003, MNRAS, 342, 837

Reid, I. N., Kirkpatrick, J. D., Liebert, J., et al. 2002, AJ, 124, 519

Scholz, R.-D., \& Meusinger, H. 2002, MNRAS, 336, L49

Scholz, R.-D., McCaughrean, M. J., Lodieu, N., \& Kuhlbrodt, B. 2003, A\&A, 398, L29

Song, I., Zuckerman, B., \& Bessel, M. S. 2003, ApJ, 599, 342 (SZB03)

Sterzik, M. F., Pascucci, I., Apai, D., et al. 2004, A\&A, 427, 245

Tinney, C. G. 1998, MNRAS, 296, L42

Webb, R. A., Zuckerman, B., Platais, I., et al. 1999, ApJ, 512, L63

Zuckerman, B., \& Song, I. 2004, ARA\&A, 42, 685

Zuckerman, B., Webb, R. A., Schwartz, M., \& Becklin, E. E. 2001, ApJ, 549, L233 